Research Article

\title{
Three-Dimensional Generalized Dynamics of Soft-Matter Quasicrystals
}

\author{
Tian-You Fan $\mathbb{D}^{1}$ and Zhi-Yi Tang $\mathbb{1 D}^{2}$ \\ ${ }^{1}$ School of Physics, Beijing Institute of Technology, Beijing 100081, China \\ ${ }^{2}$ School of Computer Science and Technology, Beijing Institute of Technology, Beijing 100081, China
}

Correspondence should be addressed to Tian-You Fan; tyfan2013@163.com

Received 19 August 2019; Accepted 10 January 2020; Published 16 April 2020

Academic Editor: Alain Portavoce

Copyright (c) 2020 Tian-You Fan and Zhi-Yi Tang. This is an open access article distributed under the Creative Commons Attribution License, which permits unrestricted use, distribution, and reproduction in any medium, provided the original work is properly cited.

The three-dimensional generalized dynamics of soft-matter quasicrystals was investigated, in which the governing equations of the dynamics are derived for observed 12-fold symmetry quasicrystals and possibly observed 8- and 10-fold symmetry ones in soft matter. The solving methods, possible solutions for some initial- and boundary-value problems of the equations, and possible applications are discussed as well.

\section{Introduction}

Fan $[1,2]$ discussed the soft-matter quasicrystals observed since 2004 [3-7], gave their dynamics equations, but only concerned the planar field. All observed soft-matter quasicrystals are two-dimensional quasicrystals so far. The two-dimensional quasicrystals consist of two kinds of ones, i.e., the first and second kinds. The first-kind ones have been studied from the three-dimensional point of view of the group representation, while the second-kind ones have been studied from the two-dimensional point of view only. Therefore, the so-called three-dimensional analysis here is given only for the first-kind ones. The three-dimensional field equations are very important and useful, for example, the flow of the soft matter past a sphere; that is, the generalized Stokes problem is significant in theory and application, and it is well known the classical Stokes solution was used by Einstein in 1905 in the Brownian motion and led to determination of Loschmidt's number and then again used by Millikan in 1922 leading to the determination of the electronic charge. In soft matter, many motions including the self-assembly of supramolecules are related to the sphere configuration, etc.

\section{Generalized Dynamics of Soft- Matter Quasicrystals}

In Refs $[1,2]$, the authors pointed out there are quite great differences between soft-matter and solid quasicrystals, from the point of view of condensed matter physics, but the formulation of the generalized hydrodynamics of the solid ones given by Lubensky et al. $[8,9]$ can be drawn for the application by the soft-matter ones, and after some modifications and supplementations, we can set up the generalized hydrodynamics, or generalized dynamics, for simplicity. The derivation of both dynamics is based on the generalized Langevin equation and the Poisson brackets, and there are many similarities to each other. However, there are main differences in principle between them:

(1) The solid viscosity constitutive equation in references [8, 9] $\sigma_{i j}^{\prime}=\eta_{i j k l} \dot{\xi}_{k l}, \dot{\xi}_{k l}=(1 / 2)\left(\left(\partial V_{k} / \partial x_{l}\right)+\left(\partial V_{l} / \partial x_{k}\right)\right)$ is replaced by the fluid constitutive equation $p_{i j}=-p \delta_{i j}+$ $\sigma_{i j}^{\prime}=-p \delta_{i j}+\eta_{i j k l} \dot{\xi}_{k l}, \sigma_{i j}^{\prime}=\eta_{i j k l} \dot{\xi}_{k l}, \quad \dot{\xi}_{k l}=(1 / 2)$ $\left(\left(\partial V_{k} / \partial x_{l}\right)+\left(\partial V_{l} / \partial x_{k}\right)\right)$ so that the constitutive laws of phonons, phasons, and fluid phonons are as follows, respectively: 


$$
\begin{aligned}
\sigma_{i j} & =C_{i j k l} \varepsilon_{i k}+R_{i j k l} w_{k l}, \\
H_{i j} & =K_{i j k l} w_{i j}+R_{k l i j} \varepsilon_{k l}, \\
p_{i j} & =-p \delta_{i j}+\sigma_{i j}^{\prime}, \sigma_{i j}^{\prime}=\eta_{i j k l} \dot{\xi}_{k l} \\
\varepsilon_{i j} & =\frac{1}{2}\left(\frac{\partial u_{i}}{\partial x_{j}}+\frac{\partial u_{j}}{\partial x_{i}}\right), \quad w_{i j}=\frac{\partial w_{i}}{\partial x_{j}} \\
\dot{\xi}_{i j} & =\frac{1}{2}\left(\frac{\partial V_{i}}{\partial x_{j}}+\frac{\partial V_{j}}{\partial x_{i}}\right)
\end{aligned}
$$

where $u_{i}$ denotes the phonon displacement vector; $\sigma_{i j}$ is the phonon stress tensor; $\varepsilon_{i j}$ is the phonon strain tensor; $w_{i}$ is the phason displacement vector; $H_{i j}$ is the phason stress tensor; $w_{i j}$ is the phason strain tensor; $V_{i}$ is the fluid phonon velocity vector; $p_{i j}$ is the fluid stress tensor; $p$ is the fluid pressure; $\eta_{i j k l}$ is the fluid viscosity coefficient tensor; $\dot{\xi}_{i j}$ is the fluid deformation rate tensor; and $C_{i j k l}, K_{i j k l}$ and $R_{i j k l}, R_{k l i j}$ are the phonon, phason, and phononphason coupling elastic constant tensors, respectively. For simplicity, we here only discuss the constitutive law of simplest fluid, i.e., $p_{i j}=-p \delta_{i j}+$ $\sigma_{i j}^{\prime}=-p \delta_{i j}+2 \eta\left(\dot{\xi}_{i j}-(1 / 3) \dot{\xi}_{k k} \delta_{i j}\right)+\eta^{\prime} \dot{\xi}_{k k}^{\prime} \delta_{i j}, \sigma_{i j}^{\prime}=$ $2 \eta\left(\dot{\xi}_{i j}-(1 / 3) \dot{\xi}_{k k} \delta_{i j}\right)+\eta^{\prime} \dot{\xi}_{k k} \delta_{i j}, \dot{\xi}_{k k}=\dot{\xi}_{11}+\dot{\xi}_{22}+\dot{\xi}_{33}$, $\dot{\xi}_{i j}=(1 / 2)\left(\left(\partial V_{i} / \partial x_{j}\right)+\left(\partial V_{j} / \partial x_{i}\right)\right)$, where $\eta$ is the first viscosity coefficient and $\eta^{\prime}$ the second one, which is omitted as it is too small (note that the description here only shows the difference of constitutive laws between the solid and the fluid; however, this does not mean that, in the solid, there is no pressure).

(2) An equation of state $p=f(\rho)$ should be supplemented, and in solid quasicrystals, an equation is unnecessary. The equation of state belongs to the thermodynamics of soft matter, so the present discussion is beyond the scope of pure hydrodynamics. We used the results from Wensink [10], with some modifications by the author [1], i.e.,

$$
p=f(\rho)=3 \frac{k_{B} T}{l^{3} \rho_{0}^{3}}\left(\rho_{0}^{2} \rho+\rho_{0} \rho^{2}+\rho^{3}\right),
$$

where $k_{B}$ is the Boltzmann constant; $T$ the absolute temperature; $l$ the characteristic size of soft matter; and $\rho_{0}$ the initial value of mass density $\rho$.

After the modification and supplementation, we obtain the governing equations of generalized dynamics of softmatter quasicrystals as follows:

$$
\begin{aligned}
\frac{\partial \rho}{\partial t}+\nabla_{k}\left(\rho V_{k}\right) & =0 \\
\frac{\partial g_{i}(r, t)}{\partial t} & =-\nabla_{k}(r)\left(V_{k} g_{i}\right)+\nabla_{j}(r)\left(-p \delta_{i j}+\eta_{i j k l} \nabla_{k}(r) V_{l}\right)-\left(\delta_{i j}-\nabla_{i} u_{j}\right) \frac{\delta H}{\delta u_{j}(r, t)}+\left(\nabla_{i} w_{j}\right) \frac{\delta H}{\delta w_{j}(r, t)}-\rho \nabla_{i}(r) \frac{\delta H}{\delta \rho(r, t)} \\
g_{j} & =\rho V_{j}, \\
\frac{\partial u_{i}(r, t)}{\partial t} & =-V_{j} \nabla_{j}(r) u_{i}-\Gamma_{u} \frac{\delta H}{\delta u_{i}(r, t)}+V_{i} \\
\frac{\partial w_{i}(r, t)}{\partial t} & =-V_{j} \nabla_{j}(r) w_{i}-\Gamma_{w} \frac{\delta H}{\delta w_{i}(r, t)}, \\
p & =f(\rho)=3 \frac{k_{B} T}{l^{3} \rho_{0}^{3}}\left(\rho_{0}^{2} \rho+\rho_{0} \rho^{2}+\rho^{3}\right)
\end{aligned}
$$


in which $H$ is the energy functional or the Hamiltonians for soft-matter quasicrystal systems. For the first kind of two-dimensional quasicrystals of soft matter, the energy functional or the Hamiltonians are similar to those given by Lubensky et al. for solid quasicrystals in the following form:

$$
\begin{aligned}
H & =H[\Psi(r, t)]=\int \frac{g^{2}}{2 \rho} d^{d} r+\int\left[\frac{1}{2} A\left(\frac{\delta \rho}{\rho_{0}}\right)^{2}+B\left(\frac{\delta \rho}{\rho_{0}}\right) \nabla \cdot u\right] d^{d} r+F_{\mathrm{el}} \\
& =H_{\text {kin }}+H_{\text {density }}+F_{\text {el }} \\
g & =\rho V, F_{\mathrm{el}}=F_{u}+F_{w}+F_{u w}
\end{aligned}
$$

where $\delta \rho=\rho-\rho_{0}, A, B$ are the material constants due to the variation of the density; $F_{\text {el }}$ denotes the elastic strain energy; and $F_{u}, F_{w}$, and $F_{u w}$ represent the strain energies of phonon coupling, phason coupling, and phonon-phason coupling of the matter, respectively:

$$
\begin{aligned}
F_{u} & =\int \frac{1}{2} C_{i j k l} \varepsilon_{i j} \varepsilon_{k l} d^{d} r, \\
F_{w} & =\int \frac{1}{2} K_{i j k l} w_{i j} w_{k l} d^{d} r, \\
F_{u w} & =\int\left(R_{i j k l} \varepsilon_{i j} w_{k l}+R_{k l i j} w_{i j} \varepsilon_{k l}\right) d^{d} r .
\end{aligned}
$$

The derivation of the first 4 equations (the equations of motion) is based on the Poisson bracket method of condensed matter physics [11], which was used by Lubensky et al. [8] in the solid quasicrystal study for the first time. The Chinese literature on this method can be found in ref. [6] and others provided by the first author, where there are many additional details on the derivations of equations of motion of quasicrystals. A key application of the Poisson bracket method lies in the Hamiltonian individual quasicrystal systems, given in the following sections. It is evident that the derivation based on Poisson brackets is not concerned about the equation of state, which is a result of thermodynamics [9].

The modified framework of hydrodynamics described by (3) is called generalized hydrodynamics, or generalized dynamics, for simplicity, which is a heritage and development of equations of Lubensky et al. created for solid quasicrystals.

\section{Soft-Matter Quasicrystals with 12- Fold Symmetry}

The discussion in Ref [6] was only concerned with the planar field and did not concern the three-dimensional problem of the dynamics, and equation (3) here holds for one, two, and three dimensions. Equation (3) is tight but very hard to understand, and it is not convenient for application in calculations.

At first, we simplify the equations for soft-matter quasicrystals with 12 -fold symmetry, which might be the most important class of soft-matter quasicrystals observed so far. For this purpose, we must list the three-dimensional constitutive laws on phonons, phasons, and fluid phonons, respectively, as follows [12-14]:

$$
\begin{aligned}
& \sigma_{x x}=C_{11} \varepsilon_{x x}+C_{12} \varepsilon_{y y}+C_{13} \varepsilon_{z z}, \\
& \sigma_{y y}=C_{12} \varepsilon_{x x}+C_{11} \varepsilon_{y y}+C_{13} \varepsilon_{z z}, \\
& \sigma_{z z}=C_{13} \varepsilon_{x x}+C_{13} \varepsilon_{y y}+C_{33} \varepsilon_{z z}, \\
& \sigma_{y z}=\sigma_{z y}=2 C_{44} \varepsilon_{y z} \\
& \sigma_{z x}=\sigma_{x z}=2 C_{44} \varepsilon_{z x} \\
& \sigma_{x y}=\sigma_{y x}=2 C_{66} \varepsilon_{x y}, \\
& H_{x x}=K_{1} w_{x x}+K_{2} w_{y y}, \\
& H_{y y}=K_{2} w_{x x}+K_{1} w_{y y}, \\
& H_{y z}=K_{4} w_{y z}, \\
& H_{x y}=\left(K_{1}+K_{2}+K_{3}\right) w_{x y}+K_{3} w_{y x} \\
& H_{x z}=K_{4} w_{x z} \\
& H_{y x}=K_{3} w_{x y}+\left(K_{1}+K_{2}+K_{3}\right) w_{y x} \\
& p_{x x}=-p+2 \eta \dot{\xi}_{x x}-\frac{2}{3} \eta \dot{\xi}_{k k}, \\
& p_{y y}=-p+2 \eta \dot{\xi}_{y y}-\frac{2}{3} \eta \dot{\xi}_{k k} \\
& p_{z z}=-p+2 \eta \dot{\xi}_{z z}-\frac{2}{3} \eta \dot{\xi}_{k k} \\
& p_{y z}=2 \eta \dot{\xi}_{y z} \\
& p_{z x}=2 \eta \dot{\xi}_{z x} \\
& p_{x y}=2 \eta \dot{\xi}_{x y} .
\end{aligned}
$$

Then, the equations of dynamics of soft-matter quasicrystals of 12-fold symmetry are given as follows by omitting the higher order terms of $\nabla_{i}\left(u_{j}\left(\delta H / \delta u_{j}\right)\right)$ and $\nabla_{i}\left(w_{j}\left(\delta H / \delta w_{j}\right)\right)$ listed in equations (3): 
$\frac{\partial \rho}{\partial t}+\nabla \cdot(\rho \mathbf{V})=0$

$$
\begin{aligned}
& \frac{\partial\left(\rho V_{x}\right)}{\partial t}+\frac{\partial\left(V_{x} \rho V_{x}\right)}{\partial x}+\frac{\partial\left(V_{y} \rho V_{x}\right)}{\partial y}+\frac{\partial\left(V_{z} \rho V_{x}\right)}{\partial z}=-\frac{\partial p}{\partial x}+\eta \nabla^{2} V_{x}+\frac{1}{3} \eta \frac{\partial}{\partial x} \nabla \cdot \mathbf{V}+\left(C_{66} \frac{\partial^{2}}{\partial y^{2}}+C_{44} \frac{\partial^{2}}{\partial z^{2}}\right) u_{x} \\
& -C_{66} \frac{\partial^{2} u_{y}}{\partial x \partial y}+\left(C_{13}+C_{44}-C_{11}\right) \frac{\partial^{2} u_{z}}{\partial x \partial z}+\left(C_{11}-B\right) \frac{\partial}{\partial x} \nabla \cdot \mathbf{u}-(A-B) \frac{1}{\rho_{0}} \frac{\partial \delta \rho}{\partial x},
\end{aligned}
$$

$$
\begin{aligned}
& \frac{\partial\left(\rho V_{y}\right)}{\partial t}+\frac{\partial\left(V_{x} \rho V_{y}\right)}{\partial x}+\frac{\partial\left(V_{y} \rho V_{y}\right)}{\partial y}+\frac{\partial\left(V_{z} \rho V_{y}\right)}{\partial z}=-\frac{\partial p}{\partial y}+\eta \nabla^{2} V_{y}+\frac{1}{3} \eta \frac{\partial}{\partial y} \nabla \cdot \mathbf{v}-C_{66} \frac{\partial^{2} u_{x}}{\partial x \partial y} \\
& +\left(C_{66} \frac{\partial^{2}}{\partial x^{2}}+C_{11} \frac{\partial^{2}}{\partial y^{2}}+C_{44} \frac{\partial^{2}}{\partial z^{2}}\right) u_{y}+\left(C_{13}+C_{44}\right) \frac{\partial^{2} u_{z}}{\partial y \partial z}+\left(C_{11}-B\right) \frac{\partial}{\partial y} \nabla \cdot \mathbf{u}-(A-B) \frac{1}{\rho_{0}} \frac{\partial \delta \rho}{\partial y},
\end{aligned}
$$$$
\frac{\partial\left(\rho V_{z}\right)}{\partial t}+\frac{\partial\left(V_{x} \rho V_{z}\right)}{\partial x}+\frac{\partial\left(V_{y} \rho V_{z}\right)}{\partial y}+\frac{\partial\left(V_{z} \rho V_{z}\right)}{\partial z}=-\frac{\partial p}{\partial z}+\eta \nabla^{2} V_{z}+\frac{1}{3} \eta \frac{\partial}{\partial z} \nabla \cdot \mathbf{V}+\left(C_{44} \frac{\partial^{2}}{\partial x^{2}}+C_{44} \frac{\partial^{2}}{\partial y^{2}}+\left(C_{33}-C_{13}-C_{44}\right) \frac{\partial^{2}}{\partial z^{2}}\right) u_{z}
$$$$
+\left(C_{13}+C_{44}-B\right) \frac{\partial}{\partial z} \nabla \cdot \mathbf{u}-(A-B) \frac{1}{\rho_{0}} \frac{\partial \delta \rho}{\partial z},
$$

$\frac{\partial u_{x}}{\partial t}+V_{x} \frac{\partial u_{x}}{\partial x}+V_{y} \frac{\partial u_{x}}{\partial y}+V_{z} \frac{\partial u_{x}}{\partial z}=V_{x}+\Gamma_{u}\left[\left(C_{11} \frac{\partial^{2}}{\partial x^{2}}+C_{66} \frac{\partial^{2}}{\partial y^{2}}+C_{44} \frac{\partial^{2}}{\partial z^{2}}\right) u_{x}+\left(C_{11}-C_{66}\right) \frac{\partial^{2} u_{y}}{\partial x \partial y}+\left(C_{13}+C_{44}\right) \frac{\partial^{2} u_{z}}{\partial x \partial z}\right]$

$\frac{\partial u_{y}}{\partial t}+V_{x} \frac{\partial u_{y}}{\partial x}+V_{y} \frac{\partial u_{y}}{\partial y}+V_{z} \frac{\partial u_{y}}{\partial z}=V_{y}+\Gamma_{u}\left[\left(C_{11}-C_{66}\right) \frac{\partial^{2} u_{x}}{\partial x \partial y}+\left(C_{66} \frac{\partial^{2}}{\partial x^{2}}+C_{11} \frac{\partial^{2}}{\partial y^{2}}+C_{44} \frac{\partial^{2}}{\partial z^{2}}\right) u_{y}+\left(C_{13}+C_{44}\right) \frac{\partial^{2} u_{z}}{\partial y \partial z}\right]$

$\frac{\partial u_{z}}{\partial t}+V_{x} \frac{\partial u_{z}}{\partial x}+V_{y} \frac{\partial u_{z}}{\partial y}+V_{z} \frac{\partial u_{z}}{\partial z}=V_{z}+\Gamma_{u}\left[\left(C_{13}+C_{44}\right)\left(\frac{\partial^{2} u_{x}}{\partial x \partial z}+\frac{\partial^{2} u_{y}}{\partial y \partial z}\right)+\left(C_{44} \frac{\partial^{2}}{\partial x^{2}}+C_{44} \frac{\partial^{2}}{\partial y^{2}}+C_{33} \frac{\partial^{2}}{\partial z^{2}}\right) u_{z}\right]$

$\frac{\partial w_{x}}{\partial t}+V_{x} \frac{\partial w_{x}}{\partial x}+V_{y} \frac{\partial w_{x}}{\partial y}+V_{z} \frac{\partial w_{x}}{\partial z}=\Gamma_{w}\left[K_{1} \nabla_{1}^{2} w_{x}+\left(K_{2}+K_{3}\right) \frac{\partial^{2} w_{x}}{\partial y^{2}}+K_{4} \frac{\partial^{2} w_{x}}{\partial z^{2}}+2 K_{3} \frac{\partial^{2} w_{y}}{\partial x \partial y}\right]$

$\frac{\partial w_{y}}{\partial t}+V_{x} \frac{\partial w_{y}}{\partial x}+V_{y} \frac{\partial w_{y}}{\partial y} V_{z} \frac{\partial w_{y}}{\partial z}=\Gamma_{w}\left[\left(K_{2}+K_{3}\right) \frac{\partial^{2} w_{x}}{\partial x \partial y}+K_{3} \frac{\partial^{2} w_{x}}{\partial y \partial z}+K_{1} \nabla_{1}^{2} w_{y}+\left(K_{2}+K_{3}\right) \frac{\partial^{2} w_{y}}{\partial x^{2}}+\left(K_{1}+K_{2}+K_{3}\right) \frac{\partial^{2} w_{y}}{\partial x \partial z}\right]$,

$p=f(\rho)=3 \frac{k_{B} T}{l^{3} \rho_{0}^{3}}\left(\rho_{0}^{2} \rho+\rho_{0} \rho^{2}+\rho^{3}\right)$, 
in which $\nabla^{2}=\left(\partial^{2} / \partial x^{2}\right)+\left(\partial^{2} / \partial y^{2}\right)+\left(\partial^{2} / \partial z^{2}\right) ; \nabla_{1}^{2}=\left(\partial^{2} /\right.$ $\left.\partial x^{2}\right)+\left(\partial^{2} / \partial y^{2}\right) ; \nabla=i(\partial / \partial x)+j(\partial / \partial y)+k(\partial / \partial z) ; \quad \mathbf{V}=$ $i V_{x}+j V_{y}+k V_{z} ; \quad \mathbf{u}=i u_{x}+j u_{y}+k u_{z} ; \quad C_{11}, C_{12}, C_{13}, C_{33}$, $C_{44}, C_{66}=\left(C_{11}-C_{12}\right) / 2$ are the phonon elastic constants; and $K_{1}, K_{2}, K_{3}, K_{4}$ are the phason elastic constants (refer to [14]); $\eta$ which is the fluid dynamic viscosity; $\Gamma_{u}$ and $\Gamma_{w}$ which are the phonon and phason dissipation coefficients; and $A$ and $B$ which are the material constants due to variation of mass density, respectively.

Equations in (7) are the final governing equations of dynamics of soft-matter quasicrystals of 12 -fold symmetry in the three-dimensional case with field variables $u_{x}, u_{y}$, $u_{z}, w_{x}, w_{y}, V_{x}, V_{y}, V_{z}, \rho$, and $p$, the amount of field variables is 10 , and the amount of field equations is 10 too; among them, (7a) is the mass conservation equation, (7b)- $(7 \mathrm{~d})$ are the momentum conservation equations, $(7 \mathrm{e})-(7 \mathrm{~g})$ are the equations of motion of phonons due to the symmetry breaking, (7h) and (7i) are the phason dissipation equations, and $(7 \mathrm{j})$ is the equation of state, respectively. The equations are consistent with mathematical solvability, and if there is lack of the equation of state, the equation system is not closed and has no meaning mathematically and physically. This shows the equation of state is necessary.

These equations reveal the nature of wave propagation of fields $\mathbf{u}$ and $\mathbf{V}$ with phonon wave speeds $c_{1}=$ $\sqrt{\left(2 A+C_{11}-B\right) / \rho}, c_{2}=c_{3}=\sqrt{\left(C_{11}-C_{12}\right) / 2 \rho}$ and fluid phonon wave speed $c_{4}=\sqrt{(\partial p / \partial \rho)_{s}}$ and the nature of the diffusion of the field $\mathbf{w}$ with the diffusive coefficient $D=1 / \Gamma_{w}$ from the view point of hydrodynamics.

\section{Soft-Matter Quasicrystals with 8- Fold Symmetry}

Apart from the observed 12- and 18-fold symmetrical softmatter quasicrystals, the 8-fold symmetrical soft-matter quasicrystals may also be observed in the near future. This kind of solid quasicrystal is very stable, which is important especially as there are strong coupling effects between the phonons and phasons, and it is interesting to study their mechanical and physical properties and mathematical solutions. We considered the plane of quasiperiodicity to be the $x y$-plane, if the $z$-axis is the 8-fold symmetry axis. Next, for the possibility of soft-matter octagonal quasicrystals in soft matter, there is the final governing equation system of the generalized dynamics, after some derivations by similar steps as in the previous section, but we must list the constitutive law [12-14] first as

$$
\begin{aligned}
& \sigma_{x x}=C_{11} \varepsilon_{x x}+C_{12} \varepsilon_{y y}+C_{13} \varepsilon_{z z}+R\left(w_{x x}+w_{y y}\right), \\
& \sigma_{y y}=C_{12} \varepsilon_{x x}+C_{11} \varepsilon_{y y}+C_{13} \varepsilon_{z z}-R\left(w_{x x}+w_{y y}\right), \\
& \sigma_{z z}=C_{13} \varepsilon_{x x}+C_{13} \varepsilon_{y y}+C_{33} \varepsilon_{z z}, \\
& \sigma_{y z}=\sigma_{z y}=2 C_{44} \varepsilon_{y z}, \\
& \sigma_{z x}=\sigma_{x z}=2 C_{44} \varepsilon_{z x}, \\
& \sigma_{x y}=\sigma_{y x}=2 C_{66} \varepsilon_{x y}-R w_{x y}+R w_{y x}, \\
& H_{x x}=K_{1} w_{x x}+K_{2} w_{y y}+R\left(\varepsilon_{x x}-\varepsilon_{y y}\right), \\
& H_{y y}=K_{2} w_{x x}+K_{1} w_{y y}+R\left(\varepsilon_{x x}-\varepsilon_{y y}\right), \\
& H_{y z}=K_{4} w_{y z}, \\
& H_{x y}=\left(K_{1}+K_{2}+K_{3}\right) w_{x y}+K_{3} w_{y x}-2 R \varepsilon_{x y}, \\
& H_{x z}=K_{4} w_{x z}, \\
& H_{y x}=K_{3} w_{x y}+\left(K_{1}+K_{2}+K_{3}\right) w_{y x}+2 R \varepsilon_{x y}, \\
& p_{x x}=-p+2 \eta \dot{\xi}_{x x}-\frac{2}{3} \eta \dot{\xi}_{k k} \\
& p_{y y}=-p+2 \eta \dot{\xi}_{y y}-\frac{2}{3} \eta \dot{\xi}_{k k} \\
& p_{z z}=-p+2 \eta \dot{\xi}_{z z}-\frac{2}{3} \eta \dot{\xi}_{k k} \\
& p_{y z}=2 \eta \dot{\xi}_{y z} \\
& p_{z x}=2 \eta \dot{\xi}_{z x} \\
& p_{x y}=2 \eta \dot{\xi}_{x y} .
\end{aligned}
$$

With these basic relations, the governing equations of three-dimensional dynamics of soft-matter quasicrystals with 8 -fold symmetry are as follows: 


$$
\begin{aligned}
& \frac{\partial \rho}{\partial t}+\nabla \cdot(\rho \mathbf{V})=0 \\
& \frac{\partial\left(\rho V_{x}\right)}{\partial t}+\frac{\partial\left(V_{x} \rho V_{x}\right)}{\partial x}+\frac{\partial\left(V_{y} \rho V_{x}\right)}{\partial y}+\frac{\partial\left(V_{z} \rho V_{x}\right)}{\partial z}=-\frac{\partial p}{\partial x}+\eta \nabla^{2} V_{x}+\frac{1}{3} \eta \frac{\partial}{\partial x} \nabla \cdot \mathbf{V}+\left(C_{66} \frac{\partial^{2}}{\partial y^{2}}+C_{44} \frac{\partial^{2}}{\partial z^{2}}\right) u_{x}+\left(C_{12}+C_{66}\right) \frac{\partial^{2} u_{y}}{\partial x \partial y} \\
& +\left(C_{13}+C_{44}-C_{11}\right) \frac{\partial^{2} u_{z}}{\partial x \partial z}+\left(C_{11}-B\right) \frac{\partial}{\partial x} \nabla \cdot \mathbf{u}+R \frac{\partial}{\partial x} \nabla_{1} \cdot \mathbf{w}-R \frac{\partial}{\partial y}\left(\frac{\partial w_{x}}{\partial y}-\frac{\partial w_{y}}{\partial x}\right)-(A-B) \frac{1}{\rho_{0}} \frac{\partial \delta \rho}{\partial x}, \\
& \frac{\partial\left(\rho V_{y}\right)}{\partial t}+\frac{\partial\left(V_{x} \rho V_{y}\right)}{\partial x}+\frac{\partial\left(V_{y} \rho V_{y}\right)}{\partial y}+\frac{\partial\left(V_{z} \rho V_{y}\right)}{\partial z}=-\frac{\partial p}{\partial y}+\eta \nabla^{2} V_{y}+\frac{1}{3} \eta \frac{\partial}{\partial y} \nabla \cdot \mathbf{V}+\left(C_{12}+C_{66}\right) \frac{\partial^{2} u_{x}}{\partial x \partial y} \\
& +\left(C_{66} \frac{\partial^{2}}{\partial x^{2}}+C_{11} \frac{\partial^{2}}{\partial y^{2}}+C_{44} \frac{\partial^{2}}{\partial z^{2}}\right) u_{y}+\left(C_{13}+C_{44}\right) \frac{\partial^{2} u_{z}}{\partial y \partial z}+\left(C_{11}-B\right) \frac{\partial}{\partial y} \nabla \cdot \mathbf{u}-R \frac{\partial}{\partial x}\left(\frac{\partial w_{x}}{\partial y}-\frac{\partial w_{y}}{\partial x}\right)-R \frac{\partial}{\partial y} \nabla_{1} \cdot \mathbf{w}-(A-B) \frac{1}{\rho_{0}} \frac{\partial \delta \rho}{\partial y}, \\
& \frac{\partial\left(\rho V_{z}\right)}{\partial t}+\frac{\partial\left(V_{x} \rho V_{z}\right)}{\partial x}+\frac{\partial\left(V_{y} \rho V_{z}\right)}{\partial y}+\frac{\partial\left(V_{z} \rho V_{z}\right)}{\partial z}=-\frac{\partial p}{\partial z}+\eta \nabla^{2} V_{z}+\frac{1}{3} \eta \frac{\partial}{\partial z} \nabla \cdot \mathbf{V} \\
& +\left(C_{44} \frac{\partial^{2}}{\partial x^{2}}+C_{44} \frac{\partial^{2}}{\partial y^{2}}+\left(C_{33}-C_{13}-C_{44}\right) \frac{\partial^{2}}{\partial z^{2}}\right) u_{z}+\left(C_{13}+C_{44}-B\right) \frac{\partial}{\partial z} \nabla \cdot \mathbf{u}-(A-B) \frac{1}{\rho_{0}} \frac{\partial \delta \rho}{\partial z}, \\
& \frac{\partial u_{x}}{\partial t}+V_{x} \frac{\partial u_{x}}{\partial x}+V_{y} \frac{\partial u_{x}}{\partial y}+V_{z} \frac{\partial u_{x}}{\partial z}=V_{x}+\Gamma_{u}\left[\left(C_{11} \frac{\partial^{2}}{\partial x^{2}}+C_{66} \frac{\partial^{2}}{\partial y^{2}}+C_{44} \frac{\partial^{2}}{\partial z^{2}}\right) u_{x}+\left(C_{12}+C_{66}\right) \frac{\partial^{2} u_{y}}{\partial x \partial y}+\left(C_{13}+C_{44}\right) \frac{\partial^{2} u_{z}}{\partial x \partial z}+R \frac{\partial}{\partial x} \nabla_{1} \cdot \mathbf{w}-R \frac{\partial}{\partial y}\left(\frac{\partial w_{x}}{\partial y}-\frac{\partial w_{y}}{\partial x}\right)\right] \\
& \frac{\partial u_{y}}{\partial t}+V_{x} \frac{\partial u_{y}}{\partial x}+V_{y} \frac{\partial u_{y}}{\partial y}+V_{z} \frac{\partial u_{y}}{\partial z}=V_{y}+\Gamma_{u}\left[\left(C_{12}+C_{66}\right) \frac{\partial^{2} u_{x}}{\partial x \partial y}+\left(C_{66} \frac{\partial^{2}}{\partial x^{2}}+C_{11} \frac{\partial^{2}}{\partial y^{2}}+C_{44} \frac{\partial^{2}}{\partial z^{2}}\right) u_{y}+\left(C_{13}+C_{44}\right) \frac{\partial^{2} u_{z}}{\partial y \partial z}-R \frac{\partial}{\partial x}\left(\frac{\partial w_{x}}{\partial y}-\frac{\partial w_{y}}{\partial x}\right)-R \frac{\partial}{\partial y} \nabla_{1} \cdot \mathbf{w}\right], \\
& \frac{\partial u_{z}}{\partial t}+V_{x} \frac{\partial u_{z}}{\partial x}+V_{y} \frac{\partial u_{z}}{\partial y}+V_{z} \frac{\partial u_{z}}{\partial z}=V_{z}+\Gamma_{u}\left[\left(C_{13}+C_{44}\right)\left(\frac{\partial^{2} u_{x}}{\partial x \partial z}+\frac{\partial^{2} u_{y}}{\partial y \partial z}\right)+\left(C_{44} \frac{\partial^{2}}{\partial x^{2}}+C_{44} \frac{\partial^{2}}{\partial y^{2}}+C_{33} \frac{\partial^{2}}{\partial z^{2}}\right) u_{z}\right] \\
& \frac{\partial w_{x}}{\partial t}+V_{x} \frac{\partial w_{x}}{\partial x}+V_{y} \frac{\partial w_{x}}{\partial y}+V_{z} \frac{\partial w_{x}}{\partial z}=\Gamma_{w}\left[K_{1} \nabla_{1}^{2} w_{x}+\left(K_{2}+K_{3}\right) \frac{\partial^{2} w_{x}}{\partial y^{2}}+K_{4} \frac{\partial^{2} w_{x}}{\partial z^{2}}+K_{2} \frac{\partial}{\partial y}\left(\frac{\partial}{\partial x}+\frac{\partial}{\partial z}\right) w_{y}+R \frac{\partial}{\partial x}\left(\frac{\partial u_{x}}{\partial x}-\frac{\partial u_{y}}{\partial y}\right)-R \frac{\partial}{\partial y}\left(\frac{\partial u_{x}}{\partial y}+\frac{\partial u_{y}}{\partial x}\right)\right] \\
& \frac{\partial w_{y}}{\partial t}+V_{x} \frac{\partial w_{y}}{\partial x}+V_{y} \frac{\partial w_{y}}{\partial y} V_{z} \frac{\partial w_{y}}{\partial z}=\Gamma_{w}\left[\left(K_{2}+K_{3}\right) \frac{\partial^{2} w_{x}}{\partial x \partial y}+K_{3} \frac{\partial^{2} w_{x}}{\partial y \partial z}+K_{1} \nabla_{1}^{2} w_{y}+\left(K_{2}+K_{3}\right) \frac{\partial^{2} w_{y}}{\partial x^{2}}+\left(K_{1}+K_{2}+K_{3}\right) \frac{\partial^{2} w_{y}}{\partial x \partial z}+R \frac{\partial}{\partial x}\left(\frac{\partial u_{x}}{\partial y}+\frac{\partial u_{y}}{\partial x}\right)+R \frac{\partial}{\partial y}\left(\frac{\partial u_{x}}{\partial x}-\frac{\partial u_{y}}{\partial y}\right)\right] \\
& p=f(\rho)=3 \frac{k_{B} T}{l^{3} \rho_{0}^{3}}\left(\rho_{0}^{2} \rho+\rho_{0} \rho^{2}+\rho^{3}\right),
\end{aligned}
$$

in which $\nabla^{2}=\left(\partial^{2} / \partial x^{2}\right)+\left(\partial^{2} / \partial y^{2}\right)+\left(\partial^{2} / \partial z^{2}\right) ; \nabla_{1}^{2}=$ $\left(\partial^{2} / \partial x^{2}\right)+\left(\partial^{2} / \partial y^{2}\right) ; \nabla=i(\partial / \partial x)+j(\partial / \partial y)+k(\partial / \partial z) ; \nabla_{1}=$ $i(\partial / \partial x)+j(\partial / \partial y) ; \quad \mathbf{V}=i V_{x}+j V_{y}+k V_{z} ; \quad \mathbf{u}=i u_{x}+$ $j u_{y}+k u_{z} ; \quad \mathbf{w}=i w_{x}+j w_{y} ; \quad C_{11}, C_{12}, C_{13}, C_{33}, C_{44}, C_{66}=$ $\left(C_{11}-C_{12}\right) / 2$ are the phonon elastic constants; $K_{1}, K_{2}, K_{3}, K_{4}$ are the phason elastic constants; $R$ is the phonon-phason coupling constant; $\eta$ is the fluid dynamic viscosity; $\Gamma_{u}$ and $\Gamma_{w}$ are the phonon and phason dissipation coefficients; and $A$ and $B$ are the material constants due to variation of mass density, respectively.

Equations in (9) are the final governing equations of dynamics of soft-matter quasicrystals of 8-fold symmetry in the three-dimensional case with field variables $u_{x}, u_{y}$, $u_{z}, w_{x}, w_{y}, V_{x}, V_{y}, V_{z}, \rho$, and $p$, the amount of field variables is 10 , and the amount of field equations is 10 too; among them, (9a) is the mass conservation equation, (9b)-(9d) are the momentum conservation equations, $(9 \mathrm{e})-(9 \mathrm{~g})$ are the equations of motion of phonons due to the symmetry breaking, (9h) and (9i) are the phason dissipation equations, and $(9 \mathrm{j})$ is the equation of state, respectively. The equations are consistent with mathematical solvability, and if there is lack of the equation of state, the equation system is not closed and has no meaning mathematically and physically. This shows the equation of state is necessary.

These equations reveal the nature of wave propagation of fields $\mathbf{u}$ and $\mathbf{V}$ with phonon wave speeds $c_{1}=$ $\sqrt{\left(2 A+C_{11}-B\right) / \rho}, c_{2}=c_{3}=\sqrt{\left(C_{11}-C_{12}\right) / 2 \rho}$ and fluid phonon wave speed $c_{4}=\sqrt{(\partial p / \partial \rho)_{s}}$ and the nature of the diffusion of the field $\mathbf{w}$ with the diffusive coefficient $D=$ $1 / \Gamma_{w}$ from the view point of hydrodynamics. 


\section{Soft-Matter Quasicrystals with 10- Fold Symmetry}

The 12 -fold symmetrical soft-matter quasicrystals were observed, with a possibility that 10 -fold symmetrical softmatter quasicrystals will be discovered thereafter. These kinds of solid quasicrystals are very stable, which promotes important interest. In particular, as there are strong coupling effects between the phonons and phasons, it is interesting to study their mechanical and physical properties and mathematical solutions. If we consider the plane in the plane to be a quasiperiodic plane, and if the $z$-axis is the 10 -fold symmetry axis, then after derivation similar to those in previous sections, we can obtain the final governing equation system for the kinds of soft-matter quasicrystals, but we must list the constitutive laws for phonons, phasons, and fluid phonons [12-14] first as

$$
\begin{aligned}
& \sigma_{x x}=C_{11} \varepsilon_{x x}+C_{12} \varepsilon_{y y}+C_{13} \varepsilon_{z z}+R\left(w_{x x}+w_{y y}\right), \\
& \sigma_{y y}=C_{12} \varepsilon_{x x}+C_{11} \varepsilon_{y y}+C_{13} \varepsilon_{z z}-R\left(w_{x x}+w_{y y}\right), \\
& \sigma_{z z}=C_{13} \varepsilon_{x x}+C_{13} \varepsilon_{y y}+C_{33} \varepsilon_{z z} \\
& \sigma_{y z}=\sigma_{z y}=2 C_{44} \varepsilon_{y z}, \\
& \sigma_{z x}=\sigma_{x z}=2 C_{44} \varepsilon_{z x}, \\
& \sigma_{x y}=\sigma_{y x}=2 C_{66} \varepsilon_{x y}-R\left(w_{x y}-w_{y x}\right), \\
& H_{x x}=K_{1} w_{x x}+K_{2} w_{y y}+R\left(\varepsilon_{x x}-\varepsilon_{y y}\right), \\
& H_{y y}=K_{2} w_{x x}+K_{1} w_{y y}+R\left(\varepsilon_{x x}-\varepsilon_{y y}\right), \\
& H_{y z}=K_{4} w_{y z}, \\
& H_{x y}=K_{1} w_{x y}-K_{2} w_{y x}, \\
& H_{x z}=K_{4} w_{x z}, \\
& H_{y x}=-K_{2} w_{x y}+K_{1} w_{y x}+2 R \varepsilon_{x y}, \\
& p_{x x}=-p+2 \eta \dot{\xi}_{x x}-\frac{2}{3} \eta \dot{\xi}_{k k}, \\
& p_{y y}=-p+2 \eta \dot{\xi}_{y y}-\frac{2}{3} \eta \dot{\xi}_{k k}, \\
& p_{z z}=-p+2 \eta \dot{\xi}_{z z}-\frac{2}{3} \eta \dot{\xi}_{k k} \\
& p_{y z}=2 \eta \dot{\xi}_{y z} \\
& p_{z x}=2 \eta \dot{\xi}_{z x} \\
& p_{x y}=2 \eta \dot{\xi}_{x y}
\end{aligned}
$$


So that we obtain the governing equations of 10 -fold symmetry quasicrystals in soft matter as

$$
\begin{aligned}
& \frac{\partial \rho}{\partial t}+\nabla \cdot(\rho \mathbf{V})=0 \\
& \frac{\partial\left(\rho V_{x}\right)}{\partial t}+\frac{\partial\left(V_{x} \rho V_{x}\right)}{\partial x}+\frac{\partial\left(V_{y} \rho V_{x}\right)}{\partial y}+\frac{\partial\left(V_{z} \rho V_{x}\right)}{\partial z}=-\frac{\partial p}{\partial x}+\eta \nabla^{2} V_{x}+\frac{1}{3} \eta \frac{\partial}{\partial x} \nabla \cdot \mathbf{V}+\left(C_{66} \frac{\partial^{2}}{\partial y^{2}}+C_{44} \frac{\partial^{2}}{\partial z^{2}}\right) u_{x} \\
& +\left(C_{12}+C_{66}\right) \frac{\partial^{2} u_{y}}{\partial x \partial y}+\left(C_{13}+C_{44}-C_{11}\right) \frac{\partial^{2} u_{z}}{\partial x \partial z}+\left(C_{11}-B\right) \frac{\partial}{\partial x} \nabla \cdot \mathbf{u}+R \frac{\partial}{\partial x} \nabla_{1} \cdot \mathbf{w}-R \frac{\partial}{\partial y}\left(\frac{\partial w_{x}}{\partial y}-\frac{\partial w_{y}}{\partial x}\right)-(A-B) \frac{1}{\rho_{0}} \frac{\partial \delta \rho}{\partial x}, \\
& \frac{\partial\left(\rho V_{y}\right)}{\partial t}+\frac{\partial\left(V_{x} \rho V_{y}\right)}{\partial x}+\frac{\partial\left(V_{y} \rho V_{y}\right)}{\partial y}+\frac{\partial\left(V_{z} \rho V_{y}\right)}{\partial z}=-\frac{\partial p}{\partial y}+\eta \nabla^{2} V_{y}+\frac{1}{3} \eta \frac{\partial}{\partial y} \nabla \cdot \mathbf{V}+\left(C_{12}+C_{66}\right) \frac{\partial^{2} u_{x}}{\partial x \partial y} \\
& +\left(C_{66} \frac{\partial^{2}}{\partial x^{2}}+C_{11} \frac{\partial^{2}}{\partial y^{2}}+C_{44} \frac{\partial^{2}}{\partial z^{2}}\right) u_{y}+\left(C_{13}+C_{44}\right) \frac{\partial^{2} u_{z}}{\partial y \partial z}+\left(C_{11}-B\right) \frac{\partial}{\partial y} \nabla \cdot \mathbf{u}-R \frac{\partial}{\partial x}\left(\frac{\partial w_{x}}{\partial y}-\frac{\partial w_{y}}{\partial x}\right)-R \frac{\partial}{\partial y} \nabla_{1} \cdot \mathbf{w}-(A-B) \frac{1}{\rho_{0}} \frac{\partial \delta \rho}{\partial y}, \\
& \frac{\partial\left(\rho V_{z}\right)}{\partial t}+\frac{\partial\left(V_{x} \rho V_{z}\right)}{\partial x}+\frac{\partial\left(V_{y} \rho V_{z}\right)}{\partial y}+\frac{\partial\left(V_{z} \rho V_{z}\right)}{\partial z}=-\frac{\partial p}{\partial z}+\eta \nabla^{2} V_{z}+\frac{1}{3} \eta \frac{\partial}{\partial z} \nabla \cdot \mathbf{V} \\
& +\left(C_{44} \frac{\partial^{2}}{\partial x^{2}}+C_{44} \frac{\partial^{2}}{\partial y^{2}}+\left(C_{33}-C_{13}-C_{44}\right) \frac{\partial^{2}}{\partial z^{2}}\right) u_{z}+\left(C_{13}+C_{44}-B\right) \frac{\partial}{\partial z} \nabla \cdot \mathbf{u}-(A-B) \frac{1}{\rho_{0}} \frac{\partial \delta \rho}{\partial z}, \\
& \frac{\partial u_{x}}{\partial t}+V_{x} \frac{\partial u_{x}}{\partial x}+V_{y} \frac{\partial u_{x}}{\partial y}+V_{z} \frac{\partial u_{x}}{\partial z}=V_{x}+\Gamma_{u}\left[\left(C_{11} \frac{\partial^{2}}{\partial x^{2}}+C_{66} \frac{\partial^{2}}{\partial y^{2}}+C_{44} \frac{\partial^{2}}{\partial z^{2}}\right) u_{x}+\left(C_{12}+C_{66}\right) \frac{\partial^{2} u_{y}}{\partial x \partial y}+\left(C_{13}+C_{44}\right) \frac{\partial^{2} u_{z}}{\partial x \partial z}+R \frac{\partial}{\partial x} \nabla_{1} \cdot \mathbf{w}-R \frac{\partial}{\partial y}\left(\frac{\partial w_{x}}{\partial y}-\frac{\partial w_{y}}{\partial x}\right)\right] \\
& \frac{\partial u_{y}}{\partial t}+V_{x} \frac{\partial u_{y}}{\partial x}+V_{y} \frac{\partial u_{y}}{\partial y}+V_{z} \frac{\partial u_{y}}{\partial z}=V_{y}+\Gamma_{u}\left[\left(C_{12}+C_{66}\right) \frac{\partial^{2} u_{x}}{\partial x \partial y}+\left(C_{66} \frac{\partial^{2}}{\partial x^{2}}+C_{11} \frac{\partial^{2}}{\partial y^{2}}+C_{44} \frac{\partial^{2}}{\partial z^{2}}\right) u_{y}+\left(C_{13}+C_{44}\right) \frac{\partial^{2} u_{z}}{\partial y \partial z}-R \frac{\partial}{\partial x}\left(\frac{\partial w_{x}}{\partial y}-\frac{\partial w_{y}}{\partial x}\right)-R \frac{\partial}{\partial y} \nabla_{1} \cdot \mathbf{w}\right] \\
& \frac{\partial u_{z}}{\partial t}+V_{x} \frac{\partial u_{z}}{\partial x}+V_{y} \frac{\partial u_{z}}{\partial y}+V_{z} \frac{\partial u_{z}}{\partial z}=V_{z}+\Gamma_{u}\left[\left(C_{13}+C_{44}\right)\left(\frac{\partial^{2} u_{x}}{\partial x \partial z}+\frac{\partial^{2} u_{y}}{\partial y \partial z}\right)+\left(C_{44} \frac{\partial^{2}}{\partial x^{2}}+C_{44} \frac{\partial^{2}}{\partial y^{2}}+C_{33} \frac{\partial^{2}}{\partial z^{2}}\right) u_{z}\right] \\
& \frac{\partial w_{x}}{\partial t}+V_{x} \frac{\partial w_{x}}{\partial x}+V_{y} \frac{\partial w_{x}}{\partial y}+V_{z} \frac{\partial w_{x}}{\partial z}=\Gamma_{w}\left[K_{1} \nabla_{1}^{2} w_{x}+K_{4} \frac{\partial^{2} w_{x}}{\partial z^{2}}+K_{2} \frac{\partial}{\partial y}\left(\frac{\partial}{\partial x}-\frac{\partial}{\partial y}\right) w_{y}+R \frac{\partial}{\partial x}\left(\frac{\partial u_{x}}{\partial x}-\frac{\partial u_{y}}{\partial y}\right)-R \frac{\partial}{\partial y}\left(\frac{\partial u_{x}}{\partial y}+\frac{\partial u_{y}}{\partial x}\right)\right] \\
& \frac{\partial w_{y}}{\partial t}+V_{x} \frac{\partial w_{y}}{\partial x}+V_{y} \frac{\partial w_{y}}{\partial y} V_{z} \frac{\partial w_{y}}{\partial z}=\Gamma_{w}\left[K_{1} \nabla_{1}^{2} w_{y}+K_{4} \frac{\partial^{2} w_{y}}{\partial z^{2}}+R \frac{\partial}{\partial x}\left(\frac{\partial u_{x}}{\partial y}+\frac{\partial u_{y}}{\partial x}\right)+R \frac{\partial}{\partial y}\left(\frac{\partial u_{x}}{\partial x}-\frac{\partial u_{y}}{\partial y}\right)\right], \\
& p=f(\rho)=3 \frac{k_{B} T}{l^{3} \rho_{0}^{3}}\left(\rho_{0}^{2} \rho+\rho_{0} \rho^{2}+\rho^{3}\right)
\end{aligned}
$$

in which $\quad \nabla^{2}=\left(\partial^{2} / \partial x^{2}\right)+\left(\partial^{2} / \partial y^{2}\right)+\left(\partial^{2} / \partial z^{2}\right) ; \nabla_{1}^{2}=$ $\left(\partial^{2} / \partial x^{2}\right)+\left(\partial^{2} / \partial y^{2}\right) ; \nabla=i(\partial / \partial x)+j(\partial / \partial y)+k(\partial / \partial z) ;$

$\mathbf{V}=i V_{x}+j V_{y}+k V_{z} ; \quad \mathbf{u}=i u_{x}+j u_{y}+k u_{z} ; \quad C_{11}, C_{12}, C_{13}$, $C_{33}, C_{44}, C_{66}=\left(C_{11}-C_{12}\right) / 2$ are the phonon elastic constants; $K_{1}, K_{2}, K_{3}, K_{4}$ are the phason elastic constants; $R$ is the phonon-phason coupling constant; $\eta$ is the fluid dynamic viscosity; $\Gamma_{u}$ and $\Gamma_{w}$ are the phonon and phason dissipation coefficients; and $A$ and $B$ are the material constants due to variation of mass density, respectively.

Equations in (11) are the final governing equations of dynamics of soft-matter quasicrystals of 10-fold symmetry in the three-dimensional case with field variables $u_{x}, u_{y}$, $u_{z}, w_{x}, w_{y}, V_{x}, V_{y}, V_{z}, \rho$, and $p$, the amount of field variables is 10 , and the amount of field equations is 10 too; among 
them, (11a) is the mass conservation equation, (11b)-(11d) are the momentum conservation equations, (11e)-(11g) are the equations of motion of phonons due to the symmetry breaking, (11h) and (11i) are the phason dissipation equations, and $(11 \mathrm{j})$ is the equation of state, respectively. The equations are consistent with mathematical solvability, and if there is lack of the equation of state, the equation system is not closed and has no meaning mathematically and physically. This shows the equation of state is necessary.

These equations reveal the nature of wave propagation of fields $\mathbf{u}$ and $\mathbf{V}$ with phonon wave speeds $c_{1}=$ $\sqrt{\left(2 A+C_{11}-B\right) / \rho}, c_{2}=c_{3}=\sqrt{\left(C_{11}-C_{12}\right) / 2 \rho}$ and fluid phonon wave speed $c_{4}=\sqrt{(\partial p / \partial \rho)_{s}}$ and the nature of the diffusion of the field $\mathbf{w}$ with the diffusive coefficient $D=$ $1 / \Gamma_{w}$ from the view point of hydrodynamics.

\section{Solving Procedure and Some Preliminary Results}

The variables $u_{x}, u_{y}, u_{z}, w_{x}, w_{y}, V_{x}, V_{y}$, and $V_{z}$ represent the fields of corresponding elementary excitations, which with fluid pressure $p$ and mass density $\rho$ follow equations in (7), (9), and (11) for different soft-matter quasicrystal systems; to determine these hydrodynamic quantities, one must solve the equations under appropriate initial and boundary conditions [15-20].

The solving of equations in (7) (or (9), or (11)) coupled with initial and boundary conditions is very difficult; the computation is very complex and can be done only by numerical methods. We used the finite difference method. The computation is stable, and this shows the equations and the formulation are correct. The preliminary results exhibit a large distinction of dynamic behaviour between soft-matter and solid quasicrystals; for example, for soft-matter quasicrystals, the compressibility $\delta \rho / \rho_{0} \sim 10^{-4}-10^{-3}$ by numerical solutions, while for solid quasicrystals, $\delta \rho / \rho_{0} \sim 10^{-13}$ in ref. [21]; meanwhile, for soft-matter quasicrystals, the ratio between fluid stress and elastic stress $p_{i j} / \sigma_{i j} \sim 1$ is shown in ref. [21], and the ratio between solid viscous stress and elastic stress $\sigma_{i}^{\prime} f \sigma_{i j} \sim 10^{-15}$ for solid quasicrystals is demonstrated in Reference [21].

As the solutions require the assistance of mathematical physics and computational physics and need a large volume for presentation, many details have not been included due to space limitations.

\section{Conclusion and Discussion}

The three-dimensional equation systems of generalized dynamics of 8-, 10-, and 12-fold symmetry two-dimensional quasicrystals in soft matter were derived. This improves the work of the first author previously for the planar field.

The solutions of the initial- and boundary-value problems of these nonlinear partial differential equations have provided fruitful results describing mass distribution, deformation, and motion of soft-matter quasicrystals, which present great differences in behaviour physically from those of solid quasicrystals.
In the theoretical system, the present dynamics is the heritage and development of the hydrodynamics of solid quasicrystals by Lubensky et al. [8, 9].

This article reports only on the three-dimensional version of field equations for the first kind of two-dimensional soft-matter quasicrystals, and it did not concern about the second kind of two-dimensional soft-matter quasicrystals, which are more complex and can be referred to [20].

More recently, ref. [22] reported a molecular dynamics modelling on possible quasicrystals of 12-fold symmetry in smectic B liquid crystals, and the work pointed out there might be a problem on the existence of phason degrees of freedom based on the observation in the modelling. This is an interesting question. In our practice $[18,21]$, the effect of phason degrees of freedom is very weak due to the decoupling between phonons and phasons in 12-fold symmetry quasicrystals based on the computation with constitutive equations in (6). However, the effect of phason degrees of freedom is very evident due to the coupling between phonons and phasons in 8 - and 10-fold symmetry quasicrystals based on our computations [21].

\section{Data Availability}

The data used to support the findings of this study are available from the corresponding author upon request.

\section{Conflicts of Interest}

The authors declare that they have no conflicts of interest.

\section{Acknowledgments}

The authors thank the National Natural Science Foundation of China for the support through Grant 11272053. Thanks are due also to Professor T. C. Lubensky at the University of Pennsylvania, USA, Professor Stephen Z. D. Cheng at the University of Akron, USA/South China University of Science and Technology, China, and Professor H. H. Wensink at the Utrecht University in the Netherlands for helpful discussions.

\section{References}

[1] T. Y. Fan, "Equation Systems of generalized hydrodynamics for soft-matter quasicrystals," Applied Mathematics and Mechanics, vol. 37, pp. 331-344, 2016, in Chinese.

[2] T. Y. Fan, "Generalized dynamics for second Kind of softmatter quasicrystals," Applied Mathemaics and Mechanics, vol. 38, pp. 189-199, 2017, in Chinese.

[3] X. Zeng, G. Ungar, Y. Liu, V. Percec, A. E. Dulcey, and J. K. Hobbs, "Supramolecular dendritic liquid quasicrystals," Nature, vol. 428, no. 6979, pp. 157-160, 2004.

[4] A. Takano, W. Kawashima, A. Noro et al., "A mesoscopic Archimedean tiling having a new complexity in an ABC star polymer," Journal of Polymer Science Part B: Polymer Physics, vol. 43, no. 18, pp. 2427-2432, 2005.

[5] D. V. Talapin, E. V. Shevchenko, M. I. Bodnarchuk, X. Ye, J. Chen, and C. B. Murray, "Quasicrystalline order in selfassembled binary nanoparticle superlattices," Nature, vol. 461, no. 7266, pp. 964-967, 2009. 
[6] S. Fischer, A. Exner, K. Zielske et al., "Colloidal quasicrystals with 12-fold and 18-fold diffraction symmetry," Proceedings of the National Academy of Sciences, vol. 108, no. 5, pp. 1810$1814,2011$.

[7] K. Yue, M. J. Huang, R. Marson et al., "Geometry induced sequence of nanoscale Frank-Kasper and quasicrystal mesophases in giant surfactants," Proceedings of the $\mathrm{Na}$ tional Academy of Sciences, vol. 113, no. 50, pp. 1932-1940, 2016.

[8] T. C. Lubensky, S. Ramaswamy, and J. Toner, "Hydrodynamics of icosahedral quasicrystals," Physical Review B, vol. 32, no. 1, pp. 7444-7452, 1985.

[9] T. C. Lubensky, Symmetry, Elasticity and Hydrodynamics of Quasicrystals, Introduction to Quasicrystals, V. M. Jaric, Ed., Academic Press, Cambridge, MA, USA, 1988.

[10] H. H. Wensink, "Equation of state of a dense columnar liquid crystal," Physical Review Letters, vol. 93, no. 15, Article ID 157801, 2004.

[11] I. E. Dzyaloshinskii and G. E. Volovick, "Poisson brackets in condensed matter physics," Annals of Physics, vol. 125, no. 1, pp. 67-97, 1980.

[12] C. Z. Hu, R. H. Wang, and D. H. Ding, "Symmetry groups, physical property tensors, elasticity and dislocations in quasicrystals," Reports on Progress in Physics, vol. 63, no. 1, pp. 1-39, 2000.

[13] T. Y. Fan, Mathematical Theory of Elasticity of Quasicrystals and its Applications, Science Press, Beijing, China/SpringerVerlag, Heidelberg,Germany, 2nd edition, 2016.

[14] T. Y. Fan, Mathematical Theory of Elasticity and Relevant Topics of Solid and Soft-Matter Quasicrystals and its Applications, Beijing Institute of Technology Press, Beijing, China, 2014, in Chinese.

[15] H. Cheng and T. Y. Fan, Flow of Soft-Matter Quasicrystals Past a Circular cylinder: Generalized Dynamics of Soft-Matter Quasicrystals, Beijing Institute of Technology Press, Beijing, China, 2019.

[16] H. Cheng, T. Y. Fan, and H. Wei, Unpublished work.

[17] F. Wang, H. Cheng, T. Y. Fan, H. Y. Hu, and Z. F. Sun, "A stress analysis of some fundamental specimens of possible soft-matter octagonal quasicrystals based on generalized dynamics," Advances in Materials Science and Engineering, vol. 2019, Article ID 8789151, 13 pages, 2019.

[18] H. Cheng, T. Y. Fan, H. Y. Hu, and Z. F. Sun, "Is the crack opened or closed in soft-matter pentagonal and decagonal quasicrystals?" Theoretical and Applied Fracture Mechanics, vol. 95, pp. 248-252, 2017.

[19] X. F. Li and T. Y. Fan, "Dislocations in the second kind twodimensional quasicrystals of soft matter," Physica B: Condensed Matter, vol. 502, pp. 175-180, 2016.

[20] T. Y. Fan, W. Yang, and X. H. Sun, "Review on generalized dynamics of soft-matter quasicrystals and its applications," 2019, https://arxiv.org/abs/1909.10676.

[21] H. Cheng, T. Y. Fan, and H. Wei, "Solutions for hydrodynamics of 5- and 10-fold symmetry quasicrystals," Applied Mathematics and Mechanics, vol. 37, no. 10, pp. 1393-1404, 2016.

[22] A. Metere, P. Oleynikov, M. Dzugutov, and S. Lidin, "A smectic dodecagonal quasicrystal," Soft Matter, vol. 12, no. 43, pp. 8869-8875, 2016. 